\title{
Trascendiendo la Dicotomía en torno a la (In) Materialidad del Patrimonio: Un Acercamiento a Partir de los Conocimientos Ecológicos Locales sobre el Uso de Vegetales
}

\author{
Transcending the Dichotomy around the (In)Material Heritage: An \\ Approach from Local Ecological Knowledge on the Use of Plants
}

Juan Martín Dabezies ${ }^{1}$

\begin{abstract}
Resumen
La crisis de la modernidad ha despertado una buisqueda de alternativas a las formas modernas de relacionamiento entre el ser humano y su medio ambiente. Además, ha generado una serie de reflexiones en relación al patrimonio cultural, otorgándole un lugar cada vez más importante a los saberes locales. En este artículo se presenta un trabajo de puesta en valor del patrimonio que se basa en el extrañamiento y la familiarizacón cultural. Para esto se documentaron y divulgaron los conocimientos asociados al manejo local de la palma Butia capitata (Sureste del Uruguay), en el marco de un programa de educación patrimonial que operó en base a la arqueologia experimental. Se discute la necesidad de deconstruir el patrimonio en valores que trasciendan su materialidad, poniendo en práctica formas de puesta en valor que articulen alteridades pasadas con alteridades presentes.
\end{abstract}

Palabras clave: Conocimientos Ecológicos, Conocimiento Local, Puesta en Valor, Patrimonio

\begin{abstract}
The crisis of modernity has generated a search for alternatives to modern forms of relationship between humans and their environment. It has also generated a series of reflections on cultural heritage, giving it an increasingly more important to local knowledge. This article presents a work of valorization of the cultural heritage based on cultural familiarization and estrangement. For this were documented and divulged the knowledge associated with the local management of the palm Butia capitata (Southeastern Uruguay), as part of a heritage education program that operated on the basis of experimental archeology. We discuss the need to deconstruct the heritage values that transcend its materiality, by implementing forms of cultural valorization that articulate past with present otherness.
\end{abstract}

Key words: Ecological Knowledge, Local Knowledge, Heritage, Valorization

\footnotetext{
Centro Universitario de la Región Este, Universidad de la República. Rincón esquina Florencio Sánchez. Rocha, Uruguay. CP 27000. Correo-e: jmdabezies@curerocha.edu.uy

Recibido: Julio 2011 Revisado: Noviembre 2011 Aceptado: Abril 2012
} 


\section{INTRODUCCIÓN}

Lo que el lector se encontrará en las páginas de este artículo es un trabajo de documentación y puesta en valor de conocimientos ecológicos locales sobre el manejo de la palma Butia capitata (Mart.) Becc. (localmente conocida como el Butiá ${ }^{1}$, en una zona rural del Sureste del Uruguay, en el departamento de Rocha. La documentación de estos saberes locales se llevó a cabo desde una perspectiva etnoarqueológica pero con una finalidad patrimonial que consistió en agregarles valor poniéndolos en acción en el marco de una serie de actividades de educación patrimonial que utilizó como herramienta principal la arqueología experimental.

A partir del registro arqueológico muchas veces es muy complejo poder entender aspectos vinculados a lo inmaterial de un artefacto utilizado en la prehistoria, como puede ser el gesto técnico, aspectos sociales y culturales de la colecta de un vegetal, las exigencias físicas, etc. Para poder hacer visibles ese tipo de cuestiones, la arqueología se ha valido de la etnoarqueología la cual tiene por finalidad realizar comparaciones con grupos similares en donde se observe un mismo fenómeno, en condiciones equivalentes al fenómeno en cuestión (Gándara 1989). Con una finalidad afín, pero para entender cuestiones más bien técnicas, la arqueología se ha valido también de la arqueología experimental, la cual consiste en replicar actividades prehistóricas para comprender otras dimensiones gracias a la práctica (Mansur 1986). Ambas disciplinas están basadas en el razonamiento por analogía, el cual puede ser definido como "the transportation of information from one objet to another on the basis of some relation of comparability between them" (Hodder 1982:16).

En este artículo se pretende dar un giro a esta orientación de la etnoarqueología y arqueología experimental como heurísticas, y utilizarlas en un marco patrimonial, adoptando un enfoque que no solo genere conocimientos a partir de los informantes, sino que los ponga en valor. En este sentido tomamos la idea de la etnoarqueología pos-procesual propuesta por Thomas (2004), en tanto que sea utilizada no para buscar similitudes, sino diferencias. Esto no significa renegar el potencial heurístico de la etnoarqueología clásica y de la arqueología experimental sobre la base del razonamiento analógico, ni pretender seguir una posición meramente narrativa ("Just So Stories") (Spriggs 2008:542), sino agregar una dimensión patrimonial a la interpretativa. 
La estrategia que seguí para incluir esta dimensión patrimonial, consistió en, por un lado, poner en valor estos conocimientos ecológicos locales sobre el procesamiento de la palma y, por otro, generar valor patrimonial a partir de un objeto arqueológico: los rompecocos. Estos artefactos son piezas líticas pasivas (es decir, no se mueven) de materias primas variables que presentan hoyuelos donde se colocan los frutos que van a ser quebrados (Figura 1) con otra pieza lítica activa que se toma con la mano.

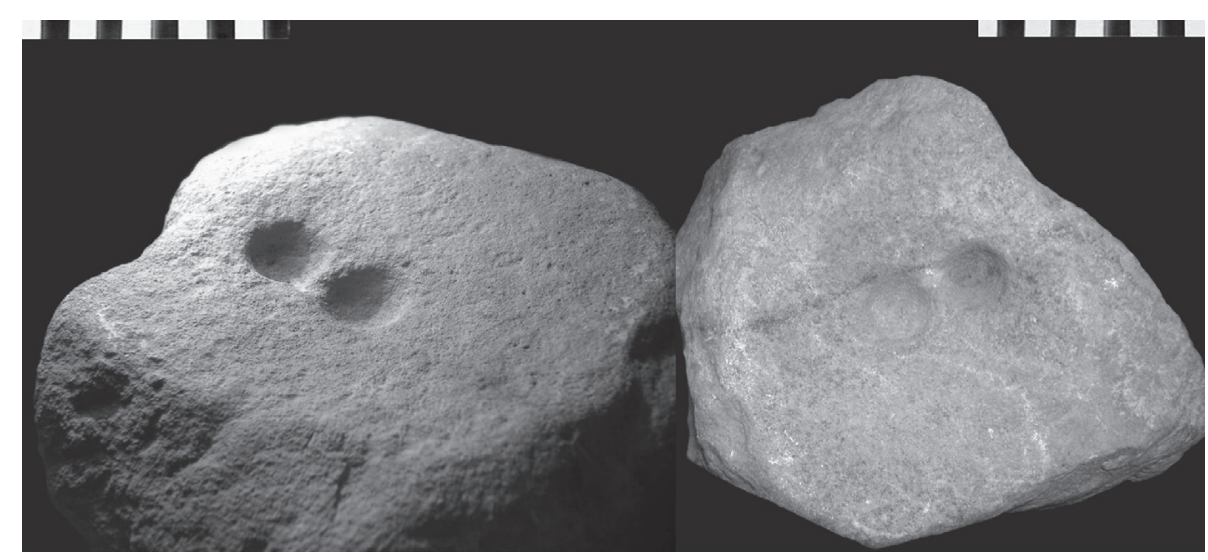

Figura 1: Dos vistas de un mismo rompecocos arqueológico. Artefacto encontrado en el túmulo llamado Los Indios, departamento de Rocha (Fuente: el autor).

Figure 1: Two views of the same archaeological rompecocos. Artifact founded in the mound called Los Indios, in the Rocha council (Source: the author).

El dispositivo metodológico que utilicé, ha sido la aplicación de un pequeño programa de educación patrimonial. Este enfoque se propone educar utilizando el patrimonio cultural como fuente primaria de conocimiento, mediante la experiencia del contacto directo con el mismo. Es un proceso activo de conocimiento en el cual se busca la apropiación y valorización del patrimonio (Horta et al. 1999).

La educación patrimonial presenta como singularidad su propuesta metodológica, en la cual se manejan recursos educacionales como el cuestionamiento constante, la experimentación y la representación (Horta 1991). Esta metodología se propone tocar puntos clave en el proceso de aprendizaje de los niños. La percepción es el primero de estos puntos; ésta se puede dividir en sensorial y significativa, según si lo que prima en la percepción son los sentidos o el significado cultural de lo que se pretende transmitir. El segundo punto es la motivación, la cual está muy relacionada 
a la percepción ya que se basa en lo concreto, lo inmediato, aspecto que puede ser despertado por la percepción. Después tenemos la capacidad de retención, que está determinada por la motivación, ya que se retiene lo que más motiva, y en el caso del niño, cuanto más concreto y perceptible mejor. Por último, pero no menos importante, está la emoción, la cual actúa de nexo entre el sentimiento y la percepción (Horta et al. 1999, Itaqui y Villagran 1998).

En este artículo se discute la posibilidad de establecer lazos entre patrimonios pasados y presentes, en base a la educación patrimonial, la etnoarqueología y la arqueología experimental. Para esto se apuesta a extrañar lo familiar y familiarizar lo extraño, proponiendo una puesta en valor que articule saberes locales y materialidades arqueológicas, en tanto referentes de alteridades pasadas y actuales.

\section{LA ZONA DE ESTUDIO}

El trabajo fue llevado a cabo en la localidad rural de Vuelta del Palmar y en la ciudad costera de La Paloma (Figura 2). Vuelta del Palmar es un poblado rural compuesto por unas 50 personas que está ubicado próximo a la ciudad de Castillos (10.000 habitantes), una de las más importantes de la zona. La Paloma es una ciudad costera próxima a la capital departamental (ciudad de Rocha, con unos 25.000 habitantes), que cuenta con unos 3.000 habitantes permanentes que ascienden a 25.000 en los meses de verano. Tanto Vuelta del Palmar como La Paloma están ubicados dentro del departamento de Rocha, en el Sureste del Uruguay. Este departamento es una región político-administrativa del Uruguay, que tiene un tipo de ocupación del territorio mayormente rural, con un modelo productivo tradicionalmente agropecuario que hace una década ha estado reorientándose al turismo (con una incidencia enorme en la faja costera). 


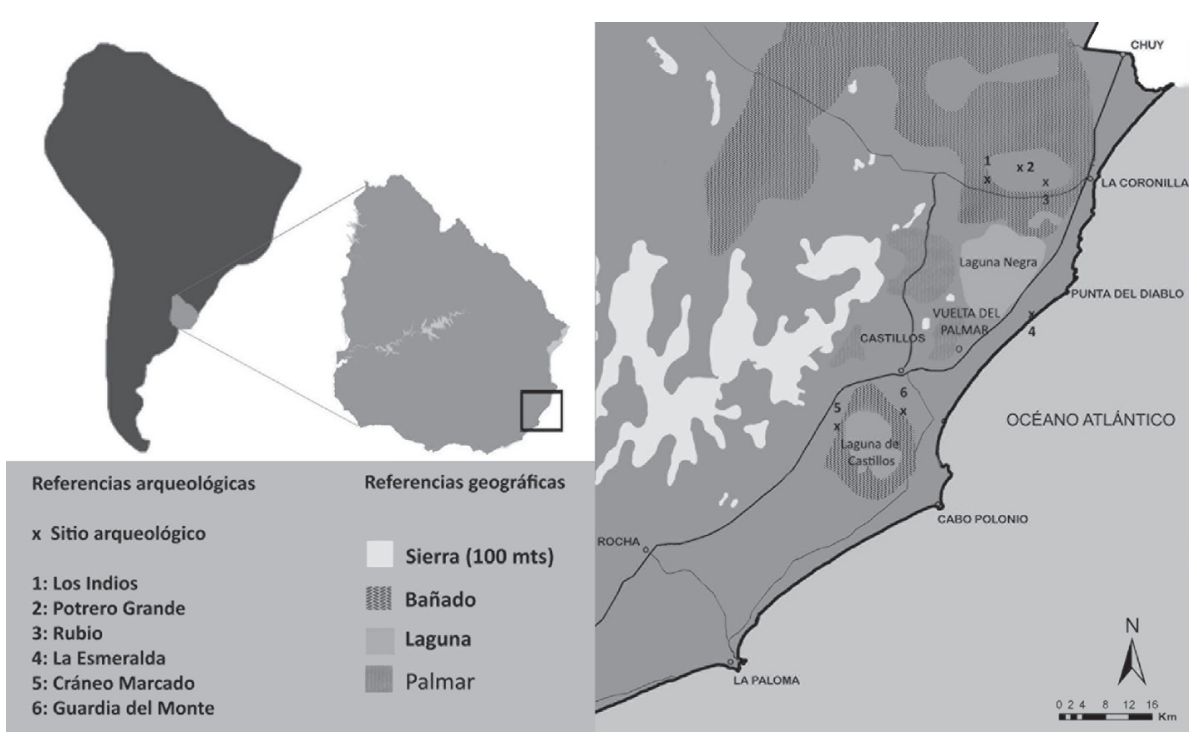

Figura 2: Ubicación de la zona de trabajo. Si bien en la zona existen más de 500 sitios arqueológicos, se indican los más importantes en cuanto a su proximidad a las localidades donde se trabajó (Fuente: el autor).

Figure 2: Location of the work. Although in the area there are over 500 archaeological sites, the map shows the most important in terms of its proximity to the locations where they worked (Source: the author).

A nivel ambiental en el departamento de Rocha coexisten sierras, lagunas, litoral atlántico, palmares y humedales, en una franja de menos de $10 \mathrm{~km}$ transversales a la costa (Figura 2). Dentro de estos ambientes se encuentran los palmares de Butia capitata, bosques de palmas que ocupan una superficie de unas 70.000 hectáreas, con densidades de hasta 600 palmas por hectárea (Rivas y Barilani 2004) (Figura 3). La palma Butia capitata es considerada como especie prioritaria para la conservación (Molina 2001, Rivas y Barilani 2004) y los palmares están protegidos bajo la figura de Reserva de la Biósfera de la UNESCO. 


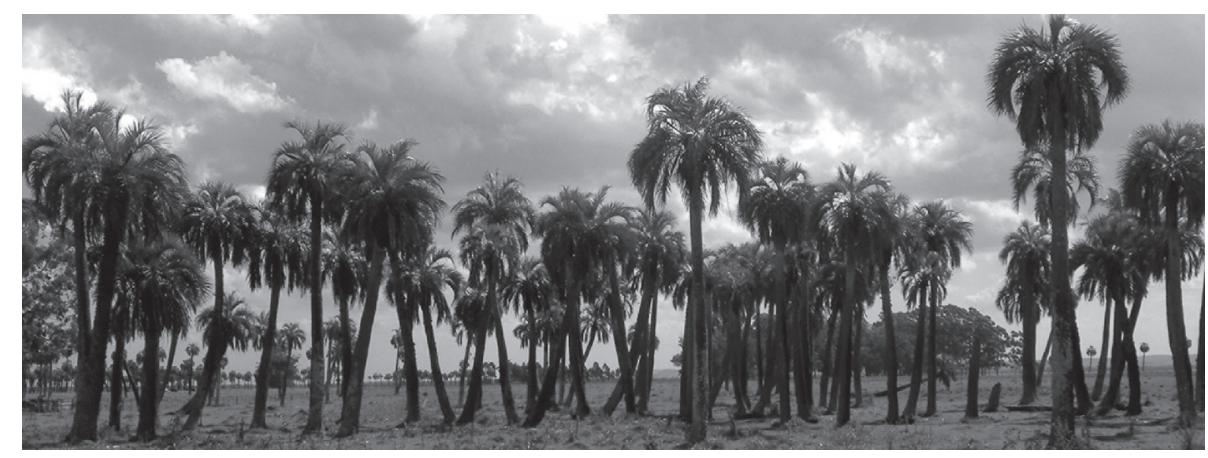

Figura 3: El palmar de Butiá capitata (Fuente: el autor).

Figure 3: The palm grove of Butia capitata (Source: the author).

La relación entre los grupos humanos de esta región y los palmares de Butia capitata data de unos 2000 años. Estos grupos indígenas, conocidos como Constructores de Cerritos, fueron caracterizados arqueológicamente por la construcción de túmulos de tierra (Cerritos de Indios) con una funcionalidad variable. Esta variabilidad funcional de tipo funeraria, monumental y doméstica, se da entre túmulos y en un mismo túmulo a lo largo del tiempo (p.e. Bracco et al. 2000, Gianotti 2000, López Mazz 2001).

Los Constructores de Cerritos poblaron esta zona desde hace algo más de 5000 años, momento en el cual eran básicamente cazadores, recolectores y pescadores. Si bien este modelo de cazador-colector dominó los paradigmas que dieron cuenta de lo indígena durante gran parte del siglo XX en América (Steward 1946), actualmente estamos en condiciones de afirmar que estos grupos fueron complejizándose a lo largo de su historia, algo que se advierte en diferentes niveles del registro arqueológico (enterramientos secundarios, especialización tecnológica, manejo de vegetales) y de la gestión del paisaje (construcción de un paisaje antrópico que controla simbólica y económicamente el territorio) (p.e. Andrade y López Mazz 2000, Cabrera 2000, Gianotti 2000, Iriarte et al. 2004, López Mazz 2000, Pintos 1999).

Dentro de estos indicadores de complejización, el manejo de los vegetales ha sido un tema abordado con mucho énfasis a partir de fines de la década de 1990, propiciado en gran medida por la mejora en las técnicas analíticas. Gracias a ello sabemos que entre el III y el II milenio antes del presente, estos grupos consumían diversos tipos de recursos vegetales, como Zea mays, Cucúrbita sp., Phaseolus sp. y Canna sp. (p.e. del Puerto e Inda 2009, del Puerto et al. 1999, Iriarte 2003, Iriarte et al. 2004). 
En este marco ha sido posible determinar que el Butiá ha tenido un lugar muy importante dentro del stock de vegetales utilizados. Existen diversas líneas de evidencias que apuntan en esta dirección: referencias etnohistóricas (César 1981, Métraux 1946, Métraux y Baldus 1946), evidencias paleobiológicas (indicadores de paleodieta y presencia de caries) (Cohe et al. 1987, Portas y Sans 1995) y paleobotánicas (presencia de micorrestos botánicos y macro-restos carbonizados) (Campos et al. 2001, del Puerto y Campos 1998). A su vez existe un indicador tecnológico que hace referencia a la utilización del fruto de esta palma. Se trata de los ya mencionados rompecocos, artefactos arqueológicos especializados en el procesamiento de los frutos. Este artefacto es también conocido en otros grupos del Paraguay y Brasil donde es llamado "quebra-nozes" o "pedras com cuvinhas" (Boretto 1980).

En lo que respecta a las relaciones en la historia reciente entre los grupos humanos y el Butiá, a mediados del siglo XX se constatan algunos usos industriales. Una de las fábricas instaladas en la zona fue Cocopalm, la cual tenía como principal cometido la extracción de aceite de palma, rompiendo los frutos secos (endocarpio) sin pulpa (mesocarpio) y luego separando la cáscara de la semilla por flotación. Otras fábricas pequeñas (con 3-5 empleados) estaban orientadas a trabajar fundamentalmente con la fibra de las hojas para elaborar suelas de calzados, cuerdas, rellenos de colchones y cojines, etc (Geymonat y Rocha 2009).

Hoy día en la región encontramos diversos tipos de personas que hacen uso directo o indirecto de la palma, del palmar y de otros recursos naturales asociados (abejas, otras especies vegetales, animales, etc.). Por ejemplo, existen artesanos/as de pequeña escala (emprendimientos familiares, cooperativas, individuales) que elaboran productos a partir del fruto, la hoja y las fibras (Figura 4); pequeños productores que llevan a cabo propuestas productivas de pastoreo, cría de animales domésticos, etc.; grandes productores con emprendimientos agrícolas, entre otros (Buffa 1992, Cardoso 1995). 

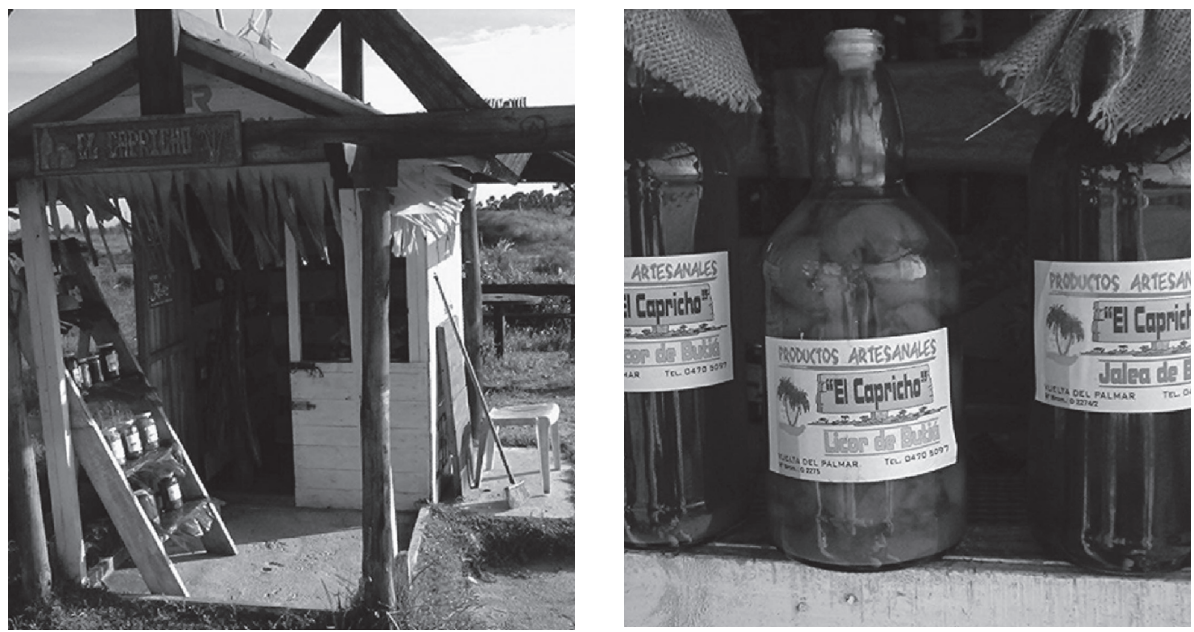

Figura 4: Venta de productos derivados del palmar de Butiá capitata en Vuelta del Palmar (Fuente: el autor).

Figure 4: Sale of products from the Butia capitata palm in Vuelta del Palmar (Source: the author).

Además, a nivel iconográfico la imagen de la palma Butiá es utilizada frecuentemente como un elemento clave de la identidad de la zona. Por ejemplo, integra el escudo departamental, es utilizada como motivo de varios murales de la ciudad de Castillos y es parte de la serie de flora autóctona de los sellos nacionales del Correo del Uruguay, entre otros. También integra textos de la literatura nacional (p.e. presente en la poesía de Carlos Roxlo), y forma parte de letras de la música popular rochense (p.e. "Caña con Butiá" de H. Ochoa y N. Pereyra) y del himno de Rocha. Es motivo de festivales artísticos locales (p.e. la celebración del "Festival de Canto y Butiá", organizado desde hace 12 años en Castillos), y tema central de publicaciones locales (Geymonat y Rocha 2009)2 ${ }^{2}$. Otro asunto interesante del uso de la imagen del Butiá, es que también es utilizada en varias logomarcas locales y nacionales (Figura 5). 

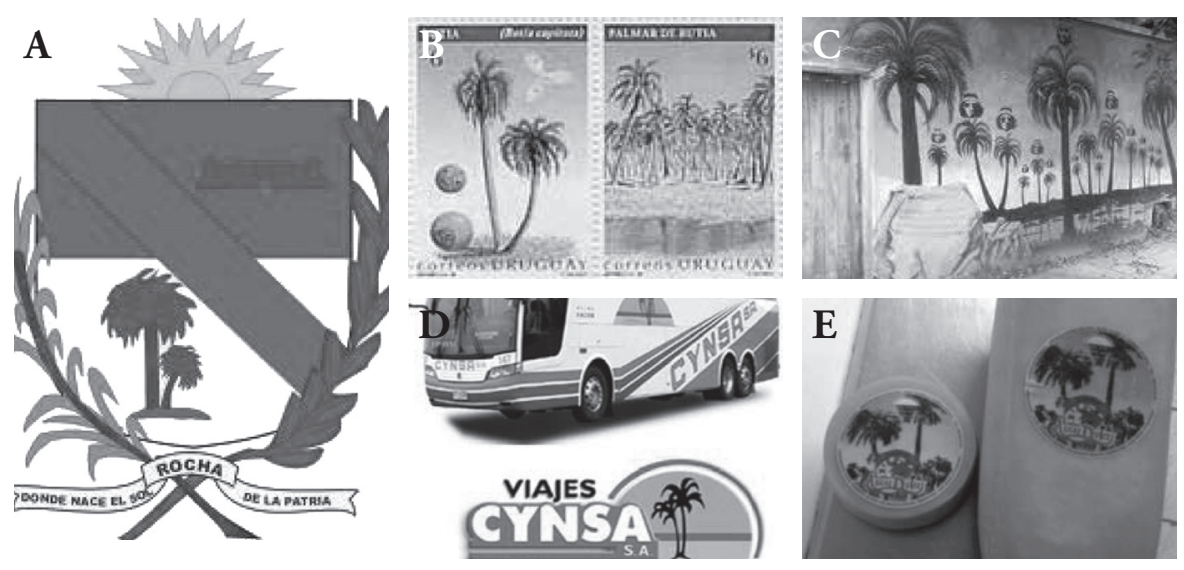

Figura 5: Usos de la iconografía del Butiá. A: escudo departamental de Rocha; B: el Butiá en los sellos nacionales; C: mural en la ciudad de Castillos; D: logomarca de empresa Cynsa (transporte de pasajeros); E: logomarcas de quesos naturales y artesanales (Montaje del autor, imágenes tomadas de diferentes fuentes on line).

Figure 5: Uses of the iconography of Butia. A: coat department of Rocha, B: Butia in national stamps, $C$ : mural in the city of Castillos; D: Cynsa company logotype (passenger transport); E: logomarks of natural and artisanal cheeses (Author's edition, images taken from different sources online).

\section{Patrimonio, identidad y CONOCIMIENTOS ECOLÓGicos LOCALES SOBRE LA PALMA BUTIA CAPITATA}

El patrimonio cultural es considerado aquí como la huella de la memoria y el olvido, una huella que,

"is comprised of all of the products of human activity and their remains, both intentional and unintentional, tangible and intangible, which represent historical and social processes, which derive their meaning from them and which, at the same time, are given a new significance by them by introducing new meanings and/or being renegotiated. This definition includes, on the one hand, objects and ideas that are socially valorized as "heritage elements", either within the context in which they are created or in another, later context; but on the other, includes the "objects" and "remains" that have the potential to achieve this valorization at a later stage due to their representative nature" (Criado-Boado et al. 2008:5). 
En este sentido, el patrimonio cultural es una construcción metacultural, algo que existe ahora pero que no existió siempre; antes había otras cosas que no eran aún patrimonio. Como proceso metacultural no existe en sí mismo, sino que depende de instancias externas a él que lo convierten en patrimonio al valorizarlo (Criado-Boado 2011).

El proceso de patrimonialización consiste en la transformación de algo que no es patrimonio en algo que sí es, o que, en el marco de este proceso es más patrimonio que antes. Este proceso de construcción del patrimonio implica una valoración en la cual intervienen distintos actores. Podemos hablar de distintos tipos de valores interrelacionados pero individualizables, como por ejemplo valor económico, científico, histórico, simbólico y estético (Ballart 1997, González Méndez 2000). En el trabajo en torno al patrimonio se generan diversas instancias de valoración con un orden no necesariamente lineal. En términos muy generales, ese orden significa que primero hay que saber qué es el patrimonio para luego poder decir qué se hace con él. Por ejemplo, primero es necesario identificarlo para luego poder protegerlo. En el marco de esta cadena valorativa se incluyen distintos momentos en la agregación de valor, como puede ser: identificación, documentación, significación, valoración, conservación, difusión y recepción (Criado-Boado 1996, González Méndez 1999, Barreiro Martínez 2003).

Uno de los procesos metaculturales más importantes, en tanto las consecuencias que tiene en el propio patrimonio, es la definición legal (Kirshenblatt-Gimblett 2004). En este sentido, la definición legal hegemónica del concepto de patrimonio cultural durante el siglo XX ha estado definida por una visión eurocéntrica, material-monumental y excepcionalista. A fines del siglo XX, se pone en tela de juicio esta concepción, el objeto único, monumental, europeo y excepcional pierde protagonismo, cediéndoselo a una concepción más amplia, mientras que paralelamente se empiezan a considerar otras dimensiones de lo material: los valores, los significados, lo intangible 3 . Es una apertura conceptual que promueve una diversidad patrimonial mucho mayor, dando cada vez más lugar a las acepciones locales (Rodríguez et al. 2003). En este marco, los saberes transmitidos oralmente en grupos no occidentales o de zonas rurales de culturas occidentales, son (re)valorados ${ }^{4}$ como una relicto de la modernidad (Touraine 1992, 1997).

Al mismo tiempo existió otro tipo de valoración vinculada a estos saberes pero en relación al discurso sobre la biodiversidad y el desarrollo sustentable (Agrawal 1995, Ellen y Harris 2000, Escobar 2000, Alexiades 2009). No existe una definición aceptada universalmente sobre qué son estos 
saberes locales 5 . En este artículo optamos por dejar de lado una terminología con fuerte carga política y moral como "tradicional" e "indígena" (Durand, 2000), refiriéndonos a conocimientos ecológicos locales (CEL de aquí en más). Estos conocimientos son considerados como "a cumulative body of knowledge, practice, and belief, evolving by adaptive processes and handed down through generations by cultural transmission, about the relationship of living beings(including humans) with one another and with their environment" (Berkes et al. 2000:1252).

La relación entre la producción científica sobre CEL y la conservación de la biodiversidad es muy marcada, a tal punto que la mayor cantidad de estudios en esta línea en Latinoamérica ocurre en los países con mayores valores de biodiversidad a nivel nacional, como es el caso de México, Colombia, Costa Rica, Venezuela y Brasil (Ruiz Müller, 2006). Uruguay no ha presentado a lo largo del siglo XX un especial interés por el estudio y protección de los CEL, en particular en el caso de los asociados a vegetales. En este sentido el campo disciplinar definido por la etnobotánica uruguaya, a escala latinoamericana es pequeño. Según Martínez (1994), en América Latina, Uruguay, Guyanas, Nicaragua y El Salvador son los países que presentan menos publicaciones en revistas internacionales sobre el tema. A su vez, junto a Paraguay, El Salvador y Puerto Rico, Uruguay es el país que presenta una menor participación en el Grupo Etnobotánico Latinoamericano (Hamilton et al. 2003).

Si bien puede llegarse a pensar que el no ser un país megadiverso puede haber incidido en el bajo interés sobre los CEL, creemos que la causa fundamental de esto radica en el proceso histórico de conformación del imaginario nacionalista de la identidad nacional uruguaya.

La identidad nacional fue forjada mirando al espejo nacionalista europeo, lo cual generó una sensación de homogeneidad cultural en donde la diferencia era sistemáticamente eliminada. Esto se ve claramente reflejado en las declaraciones oficiales publicadas en el Libro del Centenario de 1925, con motivo de la conmemoración del primer centenario de la independencia del Uruguay, en donde se decía que este país era “...la única nación de América que puede hacer la afirmación categórica de que dentro de sus límites territoriales no contiene un solo núcleo que recuerde su población aborigen" (López Campaña 1925:10). Esta lógica homogeneizante también dominó la enseñanza de la prehistoria en la educación formal uruguaya, promoviendo un trato estático, simplista y eurocéntrico de los modelos explicativos (Cabrera, 1989; Cabrera y Curbelo, 1992; Verdesio, 1992, 2000). 
Actualmente, este imaginario nacionalista, forjado a fines del siglo XIX y principios del XX (Caetano 1992), empieza a denotar una serie de fracturas generadas por los reclamos de minorías culturales vinculados a la demanda de una identidad que se mira ahora en el espejo de Latinoamérica (Porzecanski 1992). Diversos reclamos de la sociedad civil se manifiestan entre las grietas de estas fracturas. Tal es el caso del aumento de los reclamos de minorías étnicas partir de la post-dictadura (década de los 80’ y 90’) y su reconocimiento a nivel legal, como el de los afro descendientes (Frega et al, 2008).

A menor escala pero como parte de este movimiento general, en la zona de estudio se despierta un interés creciente sobre los CEL vinculados al Butiá. Se trata de un elemento vegetal que está imbricado en la identidad local, ya que fue y es parte de la economía, el arte y la cultura de la zona de estudio. A nivel nacional el interés científico y productivo manifestado en la ejecución de diversos proyectos de instituciones públicas ha tenido un importante desarrollo desde 1990 hasta la actualidad ${ }^{6}$.

A nivel local diversos tipos de emprendimientos no gubernamentales como Grupo Palmar, Eco-Palmar, Proyecto Palmares o Casa Ambiental, entre otros, que directa e indirectamente se proponen conservar y poner en valor los palmares de Butiá7. El tema fundamental de todos ellos ha sido básicamente el palmar como recurso natural, pero a partir de mediados de la década de 1990 el interés también ha estado orientado hacia los conocimientos transmitidos oralmente asociados al manejo del mismo ${ }^{8}$.

\section{DOCUMENTACIÓN DE CONOCIMIENTOS ECOLÓGICOS LOCALES Y PUESTA EN VALOR DEL PATRIMONIO}

Para comprender los usos actuales del Butiá, visité en varias ocasiones en períodos de tiempo entre 7 y 15 días durante 90 días, a habitantes de dos núcleos de productores familiares ubicados en la zona de Vuelta del Palmar. El trabajo lo llevé a cabo observando y participando ocasionalmente de las actividades domésticas y productivas, entre las cuales se encuentra el manejo del palmar. En las primeras visitas llevé a cabo varias entrevistas semidirigidas, con la finalidad de comprender los aspectos generales del uso del palmar. En las visitas finales realicé entrevistas más dirigidas, sobre la base de temas que fueron surgiendo del análisis de las entrevistas anteriores.

Tal como afirman Buffa (1992) y Cardoso (1995), en el trabajo de campo constatamos que para las familias entrevistadas el Butiá supone un 
aporte económico importante, pero siempre como complemento de otras actividades. La parte de la palma más utilizada por estos productores es el fruto, a partir del cual se elaboran jaleas, dulce de corte, caramelos, guindados, licores, etc. Los productos son vendidos mayormente en locales artesanales en los márgenes de rutas (Figura 4), aunque ahora cada vez más son incorporados en los comercios que operan en la temporada turística de verano.

Otro producto que obtienen del fruto es el localmente conocido "café de coco", el cual se elabora extrayendo la semilla del fruto. Para realizar la extracción estos productores utilizan rompecocos muy similares a los encontrados en los sitios arqueológicos de la zona. En algunas ocasiones, se han utilizado rompecocos arqueológicos encontrados en sitios superficiales (Figura 6). Para la elaboración de este producto los frutos deben estar sin pulpa, por lo que los más aptos son los digeridos y devueltos por los animales. La técnica de elaboración consiste en romper los frutos con el rompecocos y colocar el producto (cáscara y semilla) en un recipiente. En otro momento se separa la cáscara de la semilla, desechándose la primera, mientras que la segunda, rica en aceite, se deja secar y luego se tuesta, finalizando el proceso con una molienda más fina, para la cual se utiliza un molinillo pequeño similar al de café.
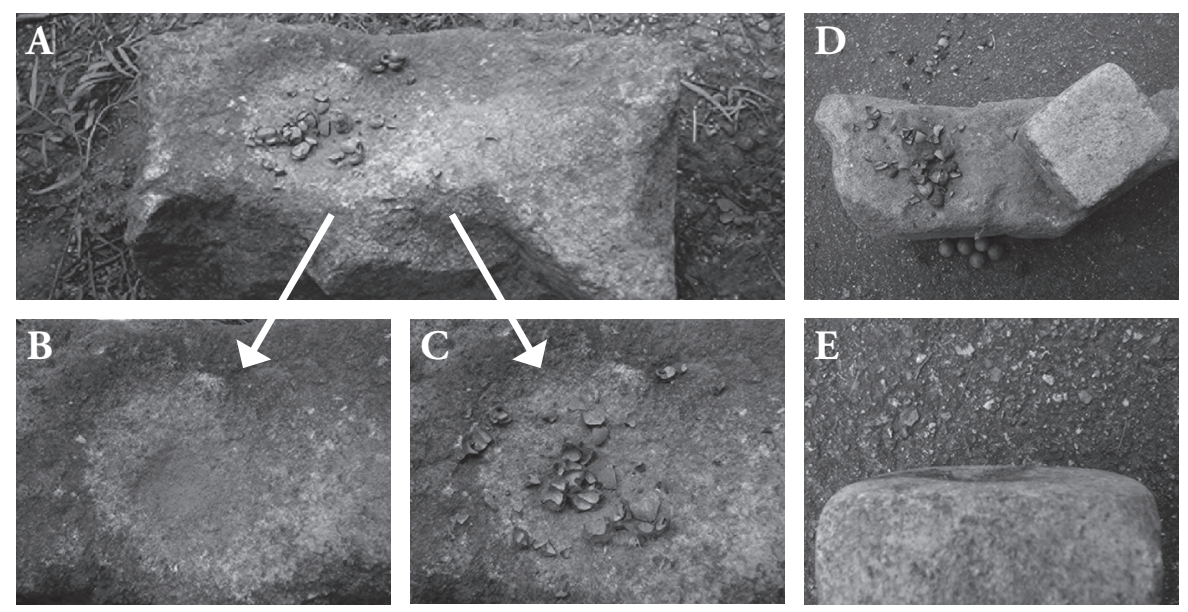

Figura 6: Molienda de los frutos secos de la palma. A: rompecocos con restos de frutos secos quebrados. $B$ y $C$ : detalles de la zona activa cóncava. D: rompecocos con mano.

E: detalle de la mano de moler (vista lateral) (Fuente: el autor).

Figure 6: Grinding the palm nuts. A: rompecocos with remnants of broken nuts. B and C: details of the concave active area. D: rompecocos with hand. E: detail of the pestle (side view) (Source: the author). 
La puesta en valor del patrimonio la llevé a cabo con talleres y exposiciones sobre la prehistoria y el patrimonio local. El objetivo de los talleres fue humanizar la prehistoria, es decir dotarla de personas, generando una subjetivación y complejización de la alteridad prehistórica. A su vez, se apuntó a la puesta en valor de los saberes locales sobre el Butiá, aumentando la profundidad temporal de la relación de los grupos humanos de la zona con el palmar. La puesta en valor de una alteridad pasada (los Constructores de Cerritos) se puso en relación con una alteridad actual (los productores de Butiá). Lo productores de Butiá no constituyen en sí mismos una alteridad para los niños, pero se generó un extrañamiento cultural basado en la inclusión de estos saberes actuales en la prehistoria.

Los talleres fueron desarrollados en cuatro jornadas consecutivas de 1,5 horas cada una, intercalándose los contenidos teóricos trabajados a modo de formulación de hipótesis para resolver situaciones problema. Esta dinámica consiste en ubicar imaginariamente a los educandos en un problema a resolver en un contexto prehistórico.

Las situaciones problema estuvieron conceptualmente organizadas en base a la oposición entre simplicidad y complejidad cultural. La simplicidad en tanto representación del paradigma del indígena cazador-recolector (Steward 1946), entendida como una otredad marginada durante más de un siglo en el proceso de construcción de la identidad nacional. La complejidad en tanto representación de los modelos interpretativos actuales, los cuales han sido parte de un proceso que ha puesto en crisis el imaginario de la identidad nacional. Esta oposición se puso en acción durante los talleres, en base al manejo de los principales argumentos en torno al estudio de la complejidad de estos grupos: uso del espacio, manejo de vegetales, tratamiento de los muertos y construcción de túmulos.

Además de este nivel más teórico de los talleres, se realizaron tres actividades prácticas que apuntaron a explotar esta línea desde una perspectiva experimental y el contacto sensorial con lo patrimonial: a) réplicas de cerámicas arqueológicas siguiendo las técnicas conocidas actualmente para la zona, b) elaboración de instrumentos de caza y c) “rompecocos". Estas actividades fueron realizadas en condiciones experimentales, es decir, respetando las condiciones materiales y tecnológicas prehistóricas, de modo que los practicantes tuvieron que activar una serie de destrezas que en su día a día son resueltas por materiales y tecnologías actuales.

Las réplicas de cerámicas arqueológicas tuvieron una parte de trabajo domiciliario, en el cual los alumnos debían procurar ellos mismos las materias primas. Una vez que los alumnos hubieron recolectado las materias 
primas, en el aula se elaboraron pequeñas vasijas de cerámica, replicando la generalidad de las formas conocidas en el registro arqueológico para la zona (cerámicas utilitarias, ocasionalmente decoradas con motivos sencillos) (Bracco et al. 2000, Capdepont 2001).

La actividad de talla experimental, fue realizada completamente en el aula, con núcleos de materias primas elaboradas por nosotros, con una mezcla de cemento portland (90\%) y arena fina (10\%). Con esta mezcla, secada al aire libre, se lograron unos núcleos blandos con buena fractura, que permitieron replicar actividades de talla experimental sin riesgos para los alumnos. Estos núcleos fueron impactados con otro lítico proporcionado por nosotros, aplicando la técnica de talla unipolar.

Las actividades "a" y "b" tuvieron por objetivo central demostrar experimentalmente la complejidad que hay detrás de la elaboración de un objeto arqueológico. Si bien no se trata de los indicadores de complejidad utilizados en el discurso arqueológico, la dinámica se orientó a complejizar algo simple en base al manejo de los saberes actuales de los alumnos en condiciones materiales que replicaron las de la alteridad prehistórica (p.e. no utilizar tecnologías no disponibles en el contexto prehistórico).

La actividad "c", consistente en manipular rompecocos para extraer la semilla de los frutos secos de las palmas, fue la más importante de la instancia de puesta en valor. Además del objetivo anterior, con esta actividad se apuntó a conectar el patrimonio arqueológico y los saberes locales actuales. Para tal actividad se utilizaron rompecocos cedidos por los productores entrevistados y fueron colectados una serie de frutos de Butiá en la zona (Figura 7).
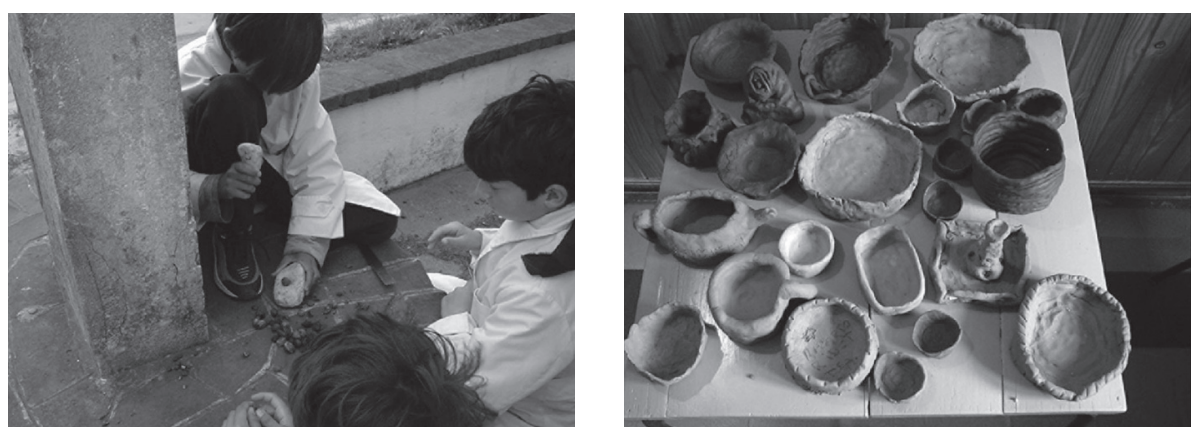

Figura 7: Actividades prácticas en los talleres. Rompiendo los frutos del Butiá con rompecocos a la izquierda y cerámicas experimentales a la derecha (Fuente: el autor). Figure 7: Practical activities in workshops. Breaking the fruits of brainteaser Butia with left and right experimental ceramic (Source: the author). 
Para finalizar la propuesta de puesta en valor, se llevaron a cabo dos exposiciones en la zona: una en el Centro Cultural de La Paloma y otra en la escuela rural de Vuelta del Palmar. La exposición de La Paloma estuvo montada durante 10 días y fue realizada previo a la de Vuelta del Palmar. Estuvo organizada intercalando paneles explicativos, dibujos de los escolares, artefactos, cerámicas y telas pintadas por ellos mismos, y materiales arqueológicos cedidos en préstamo por el Museo Nacional de Historia Natural y Antropología del Uruguay. La exposición en la escuela de Vuela del Palmar permaneció montada por 90 días. Estaba compuesta por una serie de materiales explicativos expuestos anteriormente en el Centro Cultural de La Paloma, junto con dibujos elaborados por los escolares y las cerámicas elaboradas experimentalmente con barro y arcillas locales que ellos mismos procuraron (Figura 7).

Los talleres resultaron ser las actividades más exitosas en la puesta en valor, ya que constituyeron la instancia en la cual fue posible articular de forma más efectiva los saberes locales de los niños y los de los productores, con los objetos arqueológicos, a través del contacto experimental con el objeto. Las tres actividades prácticas funcionaron como objetivadoras de los saberes que están por detrás del artefacto arqueológico, en este caso: elementos de caza, cerámicas y rompecocos. En los dos primeros casos, se apuntó más en esta dirección, mientras que en el caso del rompecocos también se pusieron en contacto los CEL pasados con los actuales, generando un mayor extrañamiento de los actuales y una mayor familiarización de los pasados.

El uso de los rompecocos locales con el mismo tipo de instrumento que utilizaban los Constructores de Cerritos resultó una actividad de transferencia de valor muy positiva. El valor histórico (al decir de Ballart, 1997) resultó ser una dimensión fundamental en el proceso de valoración patrimonial. Por otro lado, la dimensión actual otorgada por el manejo de un instrumento "vivo" y conocido, desembocó en un proceso de extrańamiento (generado por la percepción del rompecoco en tanto artefacto arqueológico) y familiarización (generado por la percepción del rompecoco en tanto herramienta utilizada por mis vecinos), que contribuyó a la patrimonialización de ambas partes (la alteridad pasada y la familiaridad actual). Además, la obtención de la semilla era un premio muy codiciado, al que todos querían acceder y degustar. De esta manera la materialidad del "coco" (semilla extraída del fruto) y la del rompecocos, generaron una fuerte sensorialidad en la actividad, la cual estuvo conectada a la dimensión patrimonial en tanto transferencia de contenidos culturales. 
En las exposiciones no fue posible lograr la misma integración entre lo inmaterial y lo material, ya que no existió un involucramiento participativo más allá del propio montaje. En La Paloma el público visitante estaba compuesto por algunos turistas y básicamente residentes del lugar, mientras que en el caso de Vuelta del Palmar los visitantes fueron básicamente los residentes de Vuelta del Palmar (varios de ellos entrevistados en las actividades de documentación), alumnos de escuelas rurales de la zona y practicantes (estudiantes de magisterio) de la región.

\section{DisCUSIÓN Y CONCLUSIONES}

Lapuestaen valordel patrimonioymásconcretamentedeestos patrimonios que durante mucho tiempo estuvieron más próximos a la huella de un olvido, son fundamentales a la hora de generar un proceso plural de construcción de las identidades colectivas. La documentación, investigación y protección de los CEL debe ser parte de un proceso de diálogo de saberes e identidades colectivas. En este marco, las actividades de puesta en valor del patrimonio utilizando como forma más común la difusión de conocimientos científicos de forma unidireccional y vertical, suele toparse con la encrucijada de la falta de articulación con contenidos dentro de la educación formal, la dificultad de ejecutar proyectos a largo plazo, de incorporarse dentro de estructuras y la dificultad del manejo de recursos y capacidades didácticas adecuadas (Dabezies 2008).

En antropología y arqueología estamos en condiciones de salvar alguna de estas dificultades, diseñando estrategias que partan de la educación patrimonial y tengan en cuenta el contacto entre las distintas dimensiones del patrimonio. En este trabajo me propuse articular estas dimensiones para transitar de la una a la otra y complementarlas. Los CEL sobre el uso del Butiá nos ayudaron a comprender mejor el uso de un artefacto arqueológico y así poder integrarlos en una estrategiaeducativa patrimonial. Pero también fue posible transitar el camino inverso y usar esos objetos arqueológicos para valorar los conocimientos locales.

La arqueología experimental puso en valor los CEL asociados a la elaboración y/o uso de artefactos morfológicamente sencillos (actividades de talla, elaboración de cerámicas, rompecocos). Esta sencillez chocó con la complejidad de su elaboración y/o uso en las condiciones experimentales. Estas actividades, enmarcadas en los talleres, pusieron en valor al objeto arqueológico en tanto producto de una gestualidad y materialidad prehistórica determinada. La arqueología experimental utilizada como herramienta de educación patrimonial, permitió trascender el objeto, llegando a los saberes que lo crean o ponen en acción. 
Para la puesta en valor de la alteridad actual (los artesanos) resultó fundamental el dotar de profundidad histórica estos CEL presentes vinculados al Butiá. El contacto entre el rompecocos, como portador de CEL prehistóricos pertenecientes a una alteridad pasada, y los CEL actuales de los artesanos, generó un efecto de valoración bidireccional. Por eso, considero que la articulación entre lo material y lo inmaterial del patrimonio, puede llevarse a cabo con una finalidad de puesta en valor, con resultados muy alentadores.

Este efecto de valoración en dos direcciones no pudo ser integrado en las exposiciones. La educación en base al patrimonio, tiene mucho más para ganar apelando a su vitalidad. En este trabajo, la puesta en acción mediante la experimentación o el registro de los CEL, tuvieron resultados mucho más alentadores que las exposiciones. Es necesario aprovechar las posibilidades que ofrece la educación patrimonial, también al momento de diseñar exposiciones. El exponer deber ser cada vez menos expositivo (en el sentido de "yo expongo y tú miras") y más participativo (“yo propongo, tú participas").

Descentrar el uso de herramientas heurísticas permite enriquecer las propias herramientas pero fundamentalmente genera nuevas formas de movernos en torno a la divulgación del patrimonio y a los propios procesos de patrimonialización. Horizontalizar métodos descentrándolos de su lugar habitual, allana el camino en la comunicación entre el saber científico y el saber local. En esta línea y en el marco de la educación patrimonial, actuaron la etnoarqueología y la arqueología experimental, acercando percepción y sensorialidad con contenidos patrimoniales.

Por último, este artículo nos permite discutir en torno a los efectos valorativos que se dan en el campo, cuando interactúan dos subjetividades en el marco de una etno-investigación. Es necesario considerar que todo registro de CEL se da en contextos de asimetrías, ya que se trata de articulaciones de al menos dos subjetividades en condiciones de desigualdad simbólica: la del investigador (portador del saber autorizado), y la del sujeto de estudio (portador del etno-saber); de hecho la propia nominación está construyendo la asimetría. Todo registro en el marco de investigaciones "etno-x" genera efectos de valoración del saber registrado. Debemos ser conscientes de los efectos que se generan al entrar al campo, algo muy discutido en antropología pero no tanto en las etno-disciplinas (etnoarqueología, entobiología, etnomusicología, etnobotánica, etnoecología, etnoentomología, etc.). En este trabajo se objetivó esta relación, con una intención patrimonializadora, que fue prevista y estratégicamente manejada al integrarse en un marco de puesta en valor. 
Agradecimientos: Fundamentalmente a las personas de Vuelta del Palmar que me abrieron las puertas de sus casas y me permitieron hacer este trabajo. También a las Escuelas involucradas y al Centro Cultural de La Paloma. A Leonel Cabrera y a José López Mazz por sus comentarios. A Néstor Pérez, Irina Capdepont, Laura del Puerto, Hugo Inda, al Museo Nacional de Antropología del Uruguay, a Carmen Curbelo, César Fagundez, Felipe Lezama, Claudio Carlé, Diego Hernández, Cecilia Pascual y Gonzalo Figueredo.

\section{Notas}

El masculino se debe a la extrapolación local del género del palmar hacia la palma. En este texto utilizaremos el masculino, siguiendo la forma local.

Cuya génesis es producto de propuestas locales. De hecho la propia publicación del libro es realizada por la ONG local Casa Ambiental con el apoyo de Misión Central Franciscana y la Unión Europea.

3 Esto puede verse claramente en la legislación internacional: la normativa sobre el patrimonio ha sufrido enormes cambios a partir de la publicación de la Convención sobre la Protección del Patrimonio Mundial Cultural y Natural (UNESCO 1972), hasta las publicaciones de informes como la Declaración Universal de la UNESCO sobre la Diversidad Cultural (UNESCO 2001a), el informe Nuestra Diversidad Creativa (UNESCO 2001b), la Declaración de Estambul (UNESCO 2002) y la Convención para la Salvaguarda del Patrimonio Cultural Inmaterial (2003), entre otros.

4 Decimos (re)valorados porque a nivel antropológico su estudio había tenido un fuerte impulso en el marco de los estudios etnocientíficos de mediados de siglo XX en Estados Unidos de América (aunque no tanto desde un punto de vista patrimonial)

De hecho ni siquiera existe una terminología única, ya que también son conocidos como conocimientos tradicionales, conocimientos ecológicos tradicionales, conocimientos ambientales tradicionales, conocimientos populares, conocimientos indígenas, conocimientos técnicos indígenas, conocimientos locales etnoecológicos, etc. (Reyes García 2009, Ellen y Haris 2000).

- Tal es el caso de proyectos liderados por el Laboratorio Tecnológico del Uruguay, la Facultad de Agronomía, Facultad de Química, Facultad de Humanidades y Ciencias de la Educación y el Programa de Conservación de la Biodiversidad y Desarrollo Sustentable en los Humedales del Este.

Ver por ejemplo el periódico local La Acción (nota del 14 de agosto del 2008, http://laaccionperiodico. blogspot.com/2008/08/nuestro-palmar-buti.html), o la web de Butiaceros, un grupo de pobladores locales que reclaman la conservación del palmar: http://butiaceros.blogspot.com/, o la web de Informe Uruguay, el artículo escrito por Julio Dornel (5 de marzo del 2010, http://www.uruguayinforme.com/ news/11072008/11072008_dornel_palmar.php)

8 A mediados de la década del 90 se generan varios videos locales (hoy colgados en la web) sobre usos tradicionales del palmar (http://cortadehojadepalma.blogspot.com/ y http://butiaceros.blogspot.com/search?updated$\min =2008-01-01$ T00\%3A00\%3A00-08\%3A00 \&updated-max $=2009-01-01 \mathrm{~T} 00 \% 3 \mathrm{~A} 00 \% 3 \mathrm{~A} 00-$ $08 \% 3 \mathrm{~A} 008$ max-results $=6$ ).

\section{BibliografíA}

Andrade, T. y J. M. López Mazz. 2000. "Emergencia de complejidad entre cazadores-recolectores del litoral Atlántico meridional sudamericano". Revista de Arqueología Americana 17(18):26-42.

Agrawal, A. 1995. "Dismantling the Divide Between Indigenous \& Scientific Knowledge". Development and Change 26(3): 413-220.

Alexiades, M. 2009. "The Cultural and Economic Globalisation of Traditional Environmental Knowledge Systems". En Landscape, Process and Power: Re- 
evaluating traditional environmental knowledge, editado por S. Heckler, pp: 68-98. Berghahn, Oxford and New York.

Ballart, J. 1997. El patrimonio histórico y arqueológico: valor y uso. Editorial Ariel, Barcelona.

Barreiro Martínez D. 2003. "Arqueología y Pragmatismo Crítico. Hacia la renovación axiológica de la Arqueología”. Claves de Razón Práctica 133: 3641.

Berkes, F., J. Colding y C. Folke. 2000. "Rediscovery of Traditional Ecological Knowledge as Adaptive Management". Ecological Applications 10(5): 12511262.

Boretto, R. 1980. Recopilación de antecedentes sobre "Piedras con Hoyuelos" de Argentina, Brasil, Chile, Paraguay y Uruguay. Museo Municipal de Historia Natural de Río Negro. Río Negro, Uruguay.

Bracco, R., L. Cabrera, y J. M. López Mazz. 2000. "La Prehistoria de las Tierras Bajas de la Cuenca de la Laguna Merin”. En Simposio Internacional de Arqueología de las Tierras Bajas, editado por A. Duran y R. Bracco, pp. 13-38. Ministerio de Educación y Cultura, Montevideo.

Buffa, V. 1992. El Palmar en la Prehistoria del Este del Uruguay. Memoria del Taller I de Arqueología. Inédito. Facultad de Humanidades y Ciencias de la Educación, Universidad de la República, Montevideo.

Cabrera, L. 1989. "El pasado que negamos". Anales del VI Encuentro Nacionaly IV Regional de Historia, pp: 58-72. Universidad de la República, Montevideo.

----- 2000. "Los Niveles de Desarrollo Socio-Cultural Alcanzados por los Grupos Constructores del Este Uruguayo". En Simposio Internacional de Arqueología de las Tierras Bajas, editado por A. Duran y R. Bracco, Pp. 169182. Ministerio de Educación y Cultura, Montevideo.

Cabrera, L. y C. Curbelo. 1992. "Patrimonio y Arqueología en el Uruguay: Hacia el reconocimiento de un Pasado Olvidado". En Arqueología en América Latina Hoy, editado por G. Politis, pp: 45-56. Banco Popular, Bogotá.

Caetano, G. 1992. "Identidad nacional e imaginario social en el Uruguay contemporáneo. La síntesis perdurable del Centenario". En La identidad Uruguaya: mito, crisis o afirmación?, editado por H. Achugar y G. Caetano, pp: 1-30. Trilce, Montevideo.

Campos, S., L. del Puerto y H. Inda. 2001.” Opal phytoliths analysis: its application to the archaeobotanical record in the East of Uruguay". En Phytoliths: Applications in Earth Sciences and Human History, editado por J. D. Maunier y F. Colin, pp: 129-142. Balkema, Lisse. 
Capdepont, I., 2001. "Análisis cerámico en la región Este del Uruguay". En Arqueología uruguaya hacia el fin del milenio, (Actas del IX Congreso Nacional de Arqueología Uruguaya), pp: 377-386. Gráficos del Sur, Montevideo.

Cardoso, L. 1995. "El palmar, la palma y el Butiá”. Fichas Didácticas de Probides 4.

César, G. 1981. Primeiros Cronistas do Río Grande do Sul. 1605-1801. Editora da UFRGS, Porto Alegre.

Cohe, R., A. Hernández y R. Bracco. 1987. "Estudio de dieta por el análisis químico de restos óseos humanos $(\mathrm{Zn}+2$ y $\mathrm{Sr}+2)$ ". Primeras Jornadas de Ciencias Antropológicas en el Uruguay, pp: 27-45. Ministerio de Educación y Cultura, Montevideo.

Criado-Boado, F. 1996. "La Arqueología del futuro, ¡el Futuro de la Arqueología?”. Trabajos de Prehistoria 53(1): 15-35.

----- 2011. El patrimonio era otra cosa. Preprint escrito para la revista "Machu Picchu". Parque Arqueológico Nacional de Machu Picchu. Cusco, Perú. Disponible en: http://hdl.handle.net/10261/32905. Fecha de acceso: 23/10/2011.

Criado-Boado, F., D. Barreiro, L. Armada, R. Blanco-Rotea, M. CostaCasais, C. Gonzalez-Perez, C. Parcero-Oubiña, P. Prieto-Martínez, y C. Sánchez-Carretero, 2008. The Heritage Laboratory Strategic Plan: An Interdisciplinary Research Line on Cultural Heritage. Laboratorio de Patrimonio-CSIC, Santiago de Compostela.

Dabezies, J. 2008. "Propuesta de inserción pragmática para una Arqueología Aplicada en Uruguay". O público e o privado 6(12): 161-174.

del Puerto, L. y H. Inda. 2009. "Estrategias de Subsistencia y Dinámica Ambiental: Análisis de silicofitolitos en sitios arqueológicos de la cuenca de Laguna de Castillos, Rocha, República Oriental del Uruguay”. En Fitolitos: estado actual de su conocimiento en América del Sur, editado por A. Zucol, M. Osterrieth, y M. Brea, Pp. 221-236. Universidad Nacional de Mar del Plata, Mar del Plata.

del Puerto, L., H. Inda y S. Campos. 1999. "Análisis de silicofitolitos de la matriz sedimentaria del sitio CG14E01, Rocha (R.O.U)”. Ameghiniana 36(4):22-39.

del Puerto, L. y S. Campos. 1998. "La recolección del Butiá: presente y pasado". En En los Tres Reinos: Prácticas de Recolección en el Cono Sur de América, editado por C. Aschero, C. Pérez, A. Korstanje y P. Vuoto, pp: 17-26. Universidad Nacional de Tucumán, Tucumán. 
Durand, L. 2000. "Modernidad y romanticismo en etnoecología". Alteridades 10(19): 143-150.

Ellen, R. y H. Harris. 2000. "Introduction". En Indigenous EnvironmentaI Knowledge and its Transformations. Critical Anthropological Perspectives, editado por R. Ellen, P. Parkes y A. Bicker, pp: 1-33. Routledge, Abingdon.

Escobar, A. 2000. "El lugar de la naturaleza y la naturaleza del lugar: ¿Globalización o Postdesarrollo?” En La colonialidad del saber: eurocentrismo y ciencias sociales perspectivas latinoamericanas, editado por E. Lander, pp: 113-143. FLACSO, Buenos Aires.

Frega, A., K., Chagas, Ó. Montańo, y N. Stalla,, 2008. "Breve historia de los afrodescendientes en el Uruguay". En Población Afrodescendiente y Desigualdades Étnico-Raciales en Uruguay, coordinado por L. Scuro, pp. 5-50. Programa de las Naciones Unidas para el Desarrollo, Montevideo.

Gándara, M. 1989. "La Analogía Etnográfica como Heurística: Lógica Muestral, Dominios Ontológicos e Historicidad". En Etnoarqueología. Coloquio Bosch-Gimpera, editado por Y. Sugiura y M. Serra, pp: 43-82. Universidad Nacional Autónoma de México, México.

Geymonat, G. y N. Rocha. 2009. Butiá-M’botia. Ecosistema único en el mundo Casa Ambiental Castillos, Uruguay.

Gianotti, C. 2000. "Paisajes Monumentales en la región Meridional Sudamericana". Gallaecia 19:43-71.

González Méndez, M. 1999. Investigación y puesta en valor del Patrimonio Histórico: planteamientos y propuestas desde la Arqueología del Paisaje. Tesis para optar al grado de Doctor en Historia. Facultad de Historia y Geografía, Universidad de Santiago de Compostela, Santiago de Compostela.

----- 2000. "Sistemas de Evaluación del Interés Patrimonial de los Yacimientos Arqueológicos". Criterios e Convencións en Arqueoloxía da Paisaxe 12:19-34.

Hamilton, A., P.Shengji, J. Kessy, A. Khan,S.Lagos-WitteyZ.Shinwari. 2003. The purposes and teaching of Applied Ethnobotany. WWF, Godalming, UK.

Hodder, I. 1982. Symbols in Actions. Cambridge University Press, Cambridge.

Horta, M. 1991. "Educaçâo Patrimonial". Conferencia Latinoamericana sobre Preservación del Patrimonio Cultural, pp: 23-55. Pontificia Universidad Católica de Porto Alegre, Porto Alegre.

Horta, M., E. Grunberg y A. Monteiro. 1999. Guía Básico de Educaçâo Patrimonial. Instituto do Patrimonio Histórico e Artístico Nacional (IPHAN), Museu Imperial., Brasilia. 
Iriarte, J. 2003. "Assessing the feasibility of identifying maize through the analysis of cross-shaped size and three-dimensional morphology of phytoliths in the grasslands of southeastern South America". Journal of Archaeological Science 30:1085-1094.

Iriarte, J., I. Holst, O. Marozzi, C. Listopard, E. Alonso, A. Rinderknecht y J. Montaña. 2004. "Evidence for cultivar adoption and emerging complexity during the mid-Holocene in the La Plata Basin". Nature 432(Diciembre):614-617.

Itaqui, J. y M. Villagran. 1998. Educaçâo Patrimonial: a experiência da quarta colonia. Universidade Federal de Santa María, Santa María.

Kirshenblatt-Gimblett, B. 2004. "El patrimonio inmaterial como producción metacultural”. Museum International 221(222): 52-67.

López Campaña, P., Ed. 1925. El Libro del Centenario del Uruguay. Ministerio de Instrucción Pública. Agencia Capurro y Cía., Montevideo.

López Mazz, J. M. 2000. “Trabajos en Tierra y Complejidad Cultural en las Tierras Bajas del Rincón de los Indios”. En Arqueología de las Tierras Bajas, editado por A. Duran y R. Bracco, Pp. 271-285. Ministerio de Educación y Cultura, Montevideo.

----- 2001. "Las estructuras tumulares (Cerritos) del Litoral Atlántico Uruguayo”. Latin American Antiquity 12(3):231-255.

Mansur, E. 1986. "Instrumentos Líticos: Aspectos da Análise Funcional". Arquivos do Museu de História Natural XI: 115-172.

Martínez, A. 1994. "Estado actual de las investigaciones etnobotánica en México”. Boletín de la Sociedad Botánica de México 55: 67-74.

Métraux, A. 1946. "The Caingang”. En Handbook of South American Indians, editado por J. Steward, pp: 445-475. Smithsonian Institution Bureau of American Ethnology Bulletin 143, Washington.

Métraux, A. y H. Baldus. 1946. "The Guayak"í. En Handbook of South American Indians, editado por J. Steward, pp: 435-444. Smithsonian Institution Bureau of American Ethnology Bulletin 143, Washington.

Molina, B., 2001. Biología y conservación del palmar de Butiá (Butia capitata) en la Reserva de Biosfera Bañados del Este. PROBIDES, Rocha.

Pintos, S. 1999. “Túmulos, caciques y otras historias. Cazadores recolectores complejos en la Cuenca de la Laguna de Castillos, Uruguay". Complutum 10:213-226. 
Portas, M. y M. Sans. 1995. "Historias de vida en los restos esqueletales de dos sitios con elevación del Dpto. de Rocha”. En Arqueología en el Uruguay: 120 años después. VIII Congreso Nacional de Arqueología Uruguaya, editado por M. Consens, J. López Mazz y C. Curbelo, pp: 32-35. Asociación Uruguaya de Arqueología. Montevideo.

Porzecanski, T. 1992. "Uruguay a fines del siglo XX: mitologías de ausencia y de presencia”. En La identidad Uruguaya: mito, crisis o afirmación?, editado por H. Achugar y G. Caetano, pp: 49-63. Trilce, Montevideo.

Reyes-García,V. 2009. "Conocimiento ecológico tradicional parala conservación: dinámicas y conflictos”. Papeles 107: 39-55.

Rivas, M. y A. Barilani. 2004. "Diversidad, potencial productivo y reproductivo de los palmares de Butia capitata (Mart.) Becc. de Uruguay”. Agrociencias VIII(1): 11-20.

Rodríguez, M. C., V. Quintero y E. Hernández. 2003. "Presentación”. En Repertorio de Textos Internacionales del Patrimonio Cultural, editado por P. Salmerón, pp: 10-13. Editorial Comares, Sevilla.

Ruiz Muller, M. 2006. La Protección Jurídica de los Conocimientos Tradicionales: Algunos Avances Politicos y Normativos en América Latina. UICN, Oficina Regional para América del Sur, Quito.

Spriggs, M. 2008. "Ethnographic parallels and the denial of history". World Archaeology 40(4): 538-552.

Steward, J. (editor) 1946. Handbook of South American Indians.1. Smithsonian Institution Bureau of American Ethnology Bulletin 143, Washington.

Thomas, J. 2004. Archaeology and Modernity. Routledge, London.

Touraine, A. 1992. Critique de la modernité. Fayard. Paris.

----- 1997. Pourrons-nous vivre ensemble ?: égaux et différents. Fayard. Paris

UNESCO. 1972. Convención sobre la Protección del Patrimonio Mundial Cultural y Natural. Paris.

----- 2001a. Declaración Universal de la UNESCO sobre la Diversidad Cultural. Paris.

---- 2001b. Nuestra Diversidad Creativa. Informe de la UNESCO, 161 EX/15. Paris.

----- 2002. Declaración de Estambul. Paris. 
Trascendiendo la Dicotomía en torno a la (In)Materialidad del Patrimonio...

----- 2003. Convención para la Salvaguardia del Patrimonio Cultural Inmaterial. Paris.

Verdesio, G. 1992. "La República Árabe Unida, el maestro soviético y la identidad nacional”. En Identidad uruguaya: ¿mito, crisis o afirmación?, editado por $\mathrm{H}$. Achugar y G. Caetano, pp: 97-107. Trilce, Montevideo.

2000. "Prehistoria de un imaginario: el territorio como escenario del drama de la diferencia”. En Uruguay: imaginarios culturales, editado por $\mathrm{H}$. Achugar y M. Moraña, pp: 11-36. Trilce. Montevideo. 